\title{
Using three-band model to retrieve chlorophyll-a of Yantai coastal waters
}

\author{
Wandong Ma ${ }^{\mathrm{a}}$, Chuanqing $\mathrm{Wu}^{\mathrm{a}}$, Shoujing Yin*a ${ }^{\mathrm{a}}$, Li Zhu ${ }^{\mathrm{a}}$, Di Wu ${ }^{\mathrm{a}}$, Qianguo Xing ${ }^{\mathrm{b}}$ \\ ${ }^{a}$ Satellite Environment Center, Ministry of Environmental Protection, Beijing China; ${ }^{b}$ Yantai \\ Institute of Coastal Zone Research, Chinese Academy of Sciences, Yantai China
}

\begin{abstract}
It is challenging that accurate assessment of chlorophyll-a concentration by remote sensing in coastal waters. Chla concentration is commonly retrieved by blue-green ratio in open ocean waters. And this method is efficient in open ocean waters. But this method is confined when applied to coastal or inland waters, because of abundant variable CDOM and tripton. It is very difficult to retrieve chla of coastal or estuary waters because of overlap of absorption and backscattering caused by CDOM and tripton. Dall'Olmo et al put forward a semi-analytical retrieval model of chla, three-band model. The conceptual three-band model has been successfully applied to estimate chla in turbid and eutrophic waters by tuning the band position in accordance with the spectral properties. The aim of this paper is to testify the three-band model that could resolve this problem. The three-band model was tuned in accord with optical properties and the bands were optimized for accurate estimation. Finally, we found a good linear relationship between chlorophyll-a and three-band model, with the determination coefficient of 0.63 and the RMSE of $2.22 \mu \mathrm{g} \cdot \mathrm{L}^{-1}$. Furthermore, the in situ spectral data was averaged to the band range of MERIS (band7, band9 and band10) and developed a simulated threeband model. A good linear relationship could be found between $\left[\left(\mathrm{B}_{7}^{-1}-\mathrm{B}_{9}{ }^{-1}\right) \times \mathrm{B}_{10}\right]$ and chlorophyll-a, with the determination coefficient of 0.59 and the RMSE of $0.72 \mu \mathrm{g} \cdot \mathrm{L}^{-1}$. The findings demonstrated that the three-band model of MERIS could be applied to retrieve chlorophyll-a concentration of Yantai coastal waters.
\end{abstract}

Keywords: Chlorophyll-a, three-band model, coastal waters, the Medium Resolution Imaging Spectrometer (MERIS)

\section{INTRODUCTION}

In case II waters, the chlorophyll-a (chla) concentration is one of the main parameters that could be retrieved from remote sensing reflectance(Rrs). Chla concentration is commonly retrieved by blue-green ratio in open ocean waters. And this method is efficient in open ocean waters. But this method is confined when applied to coastal or inland waters[1]. These algorithms that derived case I waters are not applicable to case II waters, especially for turbid and productive waters, because of abundant variable CDOM and tripton[2,, 3$]$. It is very difficult to retrieve chla of coastal or estuary waters because of overlap of absorption and backscattering caused by CDOM and tripton. In many coastal or estuary waters, the absorption of CDOM and the backscattering of tripton dominate the water column optics and influence chla retrieval. To retrieve chla concentration of coastal waters, many algorithms have been put forward, such as fluorescence algorithms, the ratio of $\operatorname{Rrs}\left(\operatorname{Rrs}_{705} / \operatorname{Rrs}_{670}\right.$ or $\left.\operatorname{Rrs}_{725} / \operatorname{Rrs}_{675}\right)$, the derivative spectrum algorithms and so on[48]. All these algorithms are based on the assumption that the absorption and fluorescence quantum yield of chla are a constant $[\underline{9}, \underline{10}]$. Actually, these parameters are variable as the environmental conditions change, such as illumination conditions, nutritional status, temperature and so on[11]. Furthermore, these empirical algorithms only could be applied in some special areas. So the chla concentration could not be retrieved accurately when using these empirical algorithms to retrieve chla concentration of coastal waters. Some researchers use analytical model for the chla retrieval. But analytical algorithms need special bio-optical parameters that could hardly be obtained for us[12]. Furthermore, the retrospective precision of these analytical algorithms is lower than that of other models. So the semi-analytical algorithms are mostly been used to retrieve chla concentration in water color remote sensing.

Recently, Dall'Olmo et al[13] put forward a semi-analytical retrieval model of chla, three-band model. The three-band model was originally used to estimate the pigments in terrestrial vegetation $[14,15]$. Afterwards, this model is applied to

*yinsj@secmep.cn; phone +81 010 58311570; fax +81 01058311501

Ocean Remote Sensing and Monitoring from Space, edited by Robert J. Frouin, Delu Pan,

Hiroshi Murakami, Young Baek Son, Proc. of SPIE Vol. 9261, 926102 · @ 2014 SPIE

CCC code: $0277-786 \mathrm{X} / 14 / \$ 18 \cdot$ doi: $10.1117 / 12.2068900$

Proc. of SPIE Vol. 9261 926102-1 
valuate chla concentration in turbid waters and furthermore was applied to the satellite data to retrieve chla concentration $[9,10,16]$. The conceptual three-band model has been successfully applied to estimate chla in turbid and eutrophic waters by tuning the band position in accordance with the spectral properties[2, $, 9,17]$. Dall'Olmo et al demonstrated that how the spectral locations of the bands $\left(\lambda_{1}, \lambda_{2}, \lambda_{3}\right)$ used affected the accuracy of chla estimation, and proposed to tune the band positions of the model minimizing the interference due to the variability in bio-optical parameters of medium and uncertainties in reflectance measurements. The estimating accuracy of chla depends on optical characteristics of the waters studied[16]. The purpose of this study is to testify the three-band model in Yantai coastal waters based on the in situ hyperspectral data.

\section{METHODOLOGY}

\subsection{Data preprocessing}

The research area lies in the south of North Yellow Sea, along the Yantai coastal line (see Fig.1). The research area, Sishili bay, is the aquaculture area and lots of feeding bait is put into it to improve fishery production every year. Thus, red tide could frequently be seen especially in summer, which could rapidly lead to the decline of fishery production. So, monitoring the status of water is an urgent task for guideline on fishery production.

A portable USB4000 spectroradiometer was used to measure above-water surface spectral reflectance in the visible and near infrared range of the spectrum. Its optical sensor could operate between $345 \mathrm{~nm}$ and $1046 \mathrm{~nm}$, with $0.2 \mathrm{~nm}$ spectral resolution in red spectral region. The spectral data were resampled with $1 \mathrm{~nm}$ spectral resolution (see Fig.2). The chla concentration was concurrently obtained. These data came from three different cruises in April, June and July, 2009, respectively.

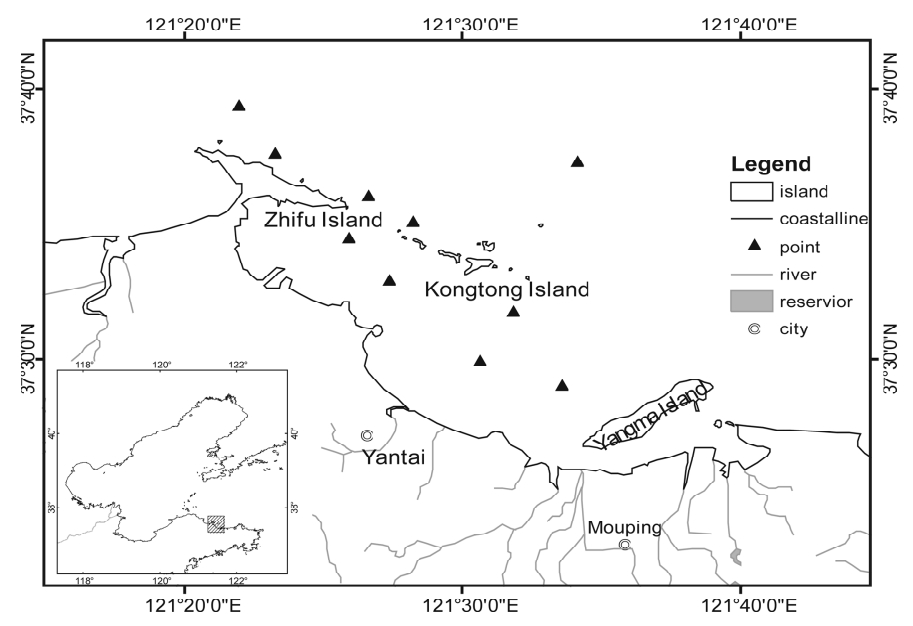

Fig.1 The distribution of sampling sites of Sishili Bay, Yantai coastal region

\subsection{Three-band model}

The three-band model is described as follows (in Eq. (1)):

$$
C_{\text {Chla }} \propto\left(R\left(\lambda_{1}\right)^{-1}-R\left(\lambda_{2}\right)^{-1}\right) R\left(\lambda_{3}\right)
$$

Where $C_{\text {Chla }}$ is the concentration of chla; $R\left(\lambda_{1}\right), R\left(\lambda_{2}\right)$ and $R\left(\lambda_{3}\right)$ are the Rrs at $\lambda_{1}, \lambda_{2}, \lambda_{3}$ wavelength, respectively. The wavelengths in three-band model are selected according to the inherent optical properties (IOP) of chla: the absorption and backscattering coefficients[9, 10]. Based on the curves of Rrs (see Fig.2) and the principle of three-band model, the wavelength of $670 \mathrm{~nm}, 690 \mathrm{~nm}$ and $730 \mathrm{~nm}$ were selected as $\lambda_{1}, \lambda_{2}$ and $\lambda_{3}$, respectively, so as to build three-band model. The three-band model was built according to the selected bands (see Fig.3) The determination coefficient of the threeband model was 0.63 , and the root mean square error (RMSE) was $2.22 \mu \mathrm{g} \cdot \mathrm{L}^{-1}$. 
According to the principal of three-band model and band set of MERIS, the ranges of the three bands (band7, band9 and band10) were selected and they were $660 \sim 670 \mathrm{~nm}, 704 \sim 714 \mathrm{~nm}, 750 \sim 758 \mathrm{~nm}$, respectively. The spectral data in situ measurements were averaged to these band ranges.

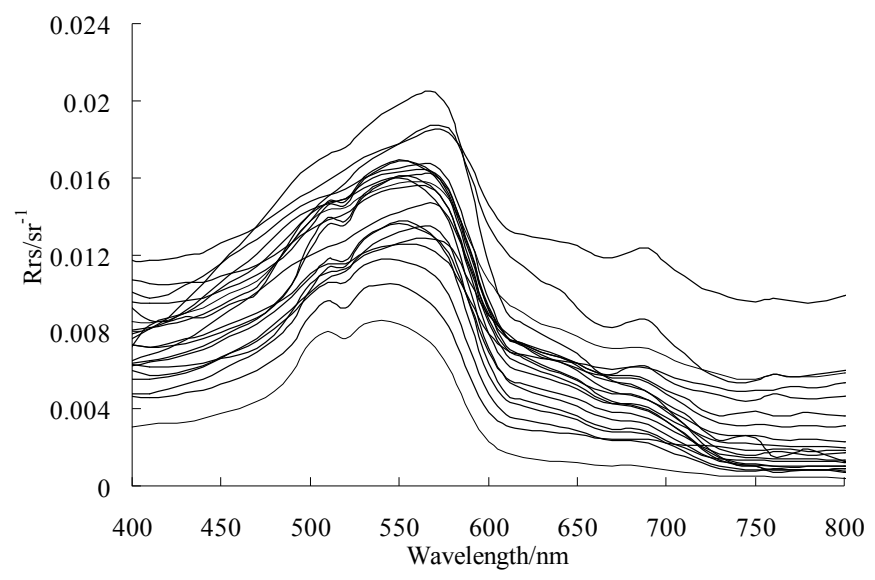

Fig.2 Spectral reflectance curves of Sishili Bay

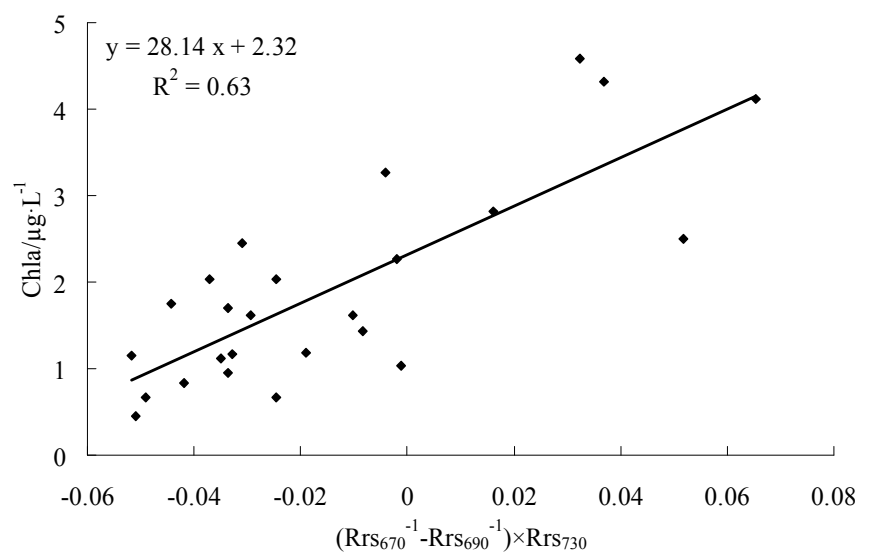

Fig.3 Relationship between chla and $\left[\left(\mathrm{R}_{670^{-1}}-\mathrm{Rrs}_{690}{ }^{-1}\right) \times \operatorname{Rrs}_{730}\right]$

The three-band model between the averaged spectral data and chla concentration was built (see Fig.4). The determination coefficient of MERIS three-band model is 0.59 and the RMSE is $0.72 \mu \mathrm{g} \cdot \mathrm{L}^{-1}$ when used to retrieve chla concentration.

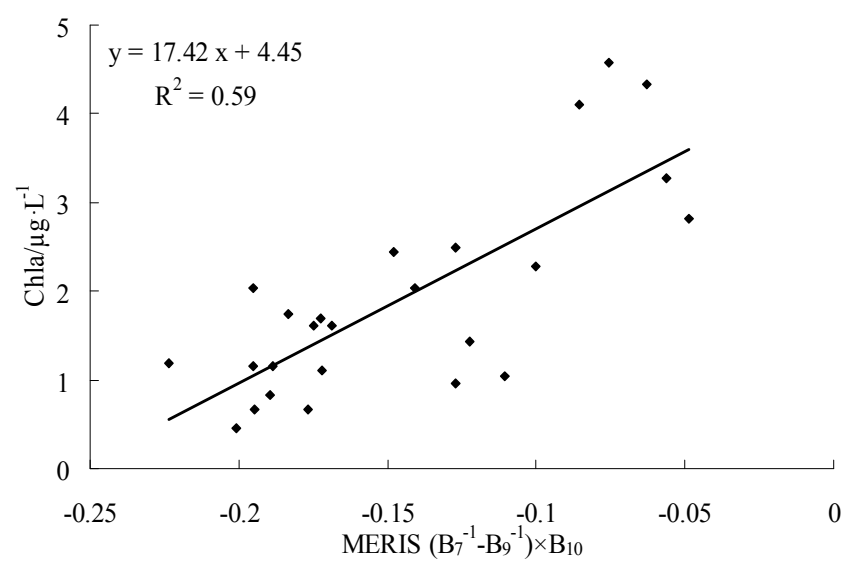

Fig.4 Relationship between chla and MERIS $\left[\left(\mathrm{B}_{7}^{-1}-\mathrm{B}_{9}{ }^{-1}\right) \times \mathrm{B}_{10}\right]$ 


\section{DISCUSSION}

We applied other algorithms in order to validate the retrieval precision of three-band model. Dall'Olmo et al found the band ratio of $\operatorname{Rrs}_{725} / \operatorname{Rrs}_{675}$ had a relative good correlation with chla concentration[2, $\underline{8}, \underline{18}, \underline{19}$ ]. So the band ratio of $\operatorname{Rrs}_{725} / \operatorname{Rrs}_{675}$ was used to retrieve chla concentration in this study. The determination coefficient of the linear relationship between the ratio of $\operatorname{Rrs}_{725} / \operatorname{Rrs}_{675}$ and chla, is 0.36 , with the RMSE of $0.90 \mu \mathrm{g} \cdot \mathrm{L}^{-1}$ (see Fig.5(a)). The fluorescence line is commonly used to retrieve chla concentration[4, 20, 21]. In this study, $\operatorname{Rrs}_{690}$ is the peak of chla fluorescence and the linear model between the $\operatorname{Rrs}_{690}$ and chla concentration is built according Fig.1. The determination coefficient of the linear relationship between the $\operatorname{Rrs}_{690}$ and chla is 0.48 , with the RMSE of $0.43 \mu \mathrm{g} \cdot \mathrm{L}^{-1}$ (see Fig.5(b)). The fluorescence line height(FLH) is also applied to retrieve chla concentration in this paper. And the linear relationship is not obvious, with determination coefficient of 0.22 and the RMSE of $1.0 \mu \mathrm{g} \cdot \mathrm{L}^{-1}$ (see Fig.5(c)).
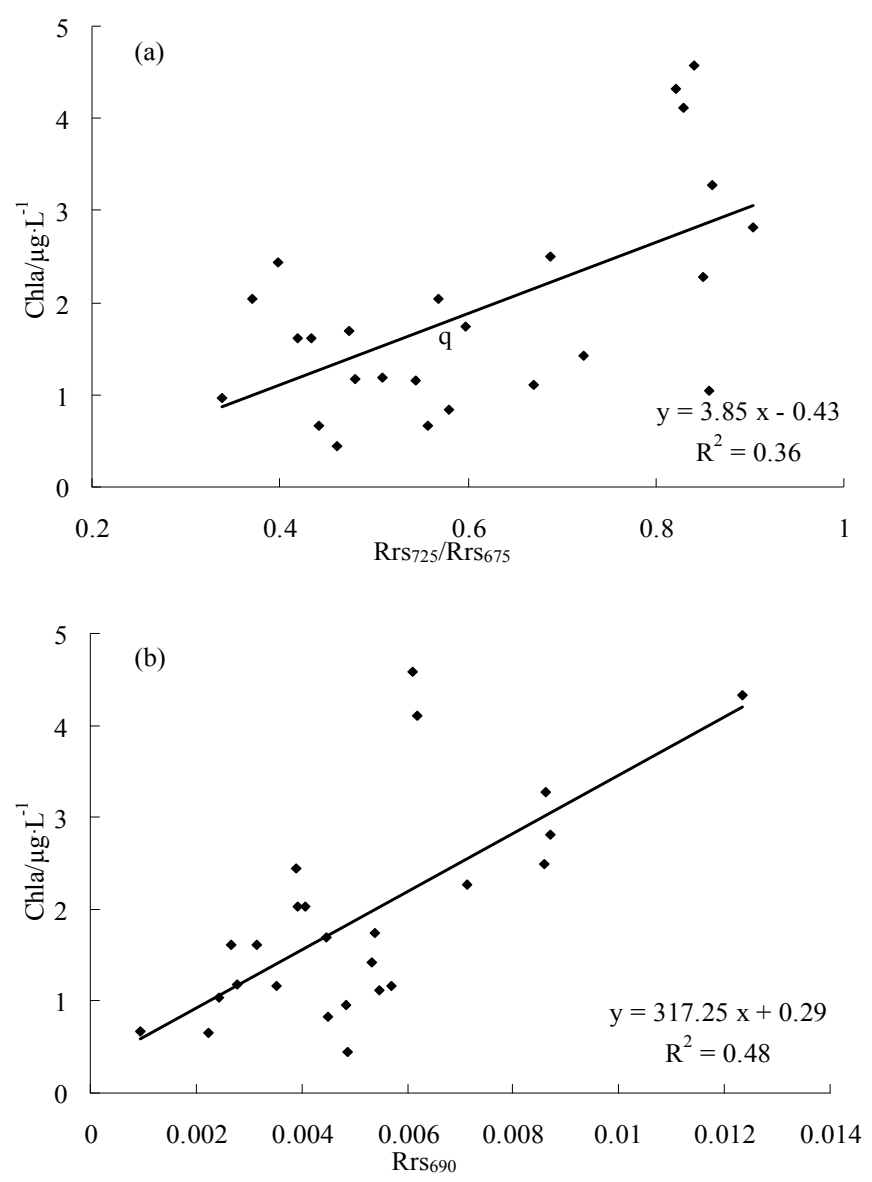


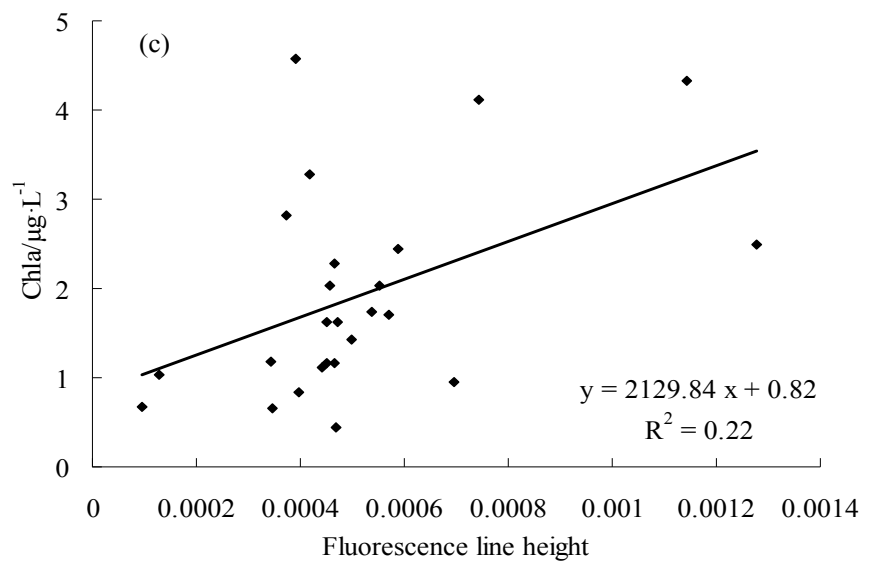

Fig.5 Several retrieval algorithms of chla concentration

The biases of different models were also compared in table 1. In summary, from the determination coefficient, RMSE and bias of different models, we could conclude that the three-band model could be applied to retrieve chla concentration in our research areas..

Table 1 Comparison of different retrieval algorithms of chla

\begin{tabular}{|c|c|c|c|}
\hline Retrieval algorithms & $\mathbf{R}^{2}$ & $\begin{array}{l}\text { RMSE } \\
\left(\mu \mathrm{g} \cdot \mathrm{L}^{-1}\right)\end{array}$ & $\begin{array}{c}\text { Bias } \\
\left(\mu \mathrm{g} \cdot \mathbf{L}^{-1}\right)\end{array}$ \\
\hline$\left(\operatorname{Rrs}_{670^{-1}}-\operatorname{Rrs}_{690}{ }^{-1}\right) \times \operatorname{Rrs}_{730}$ & 0.63 & 2.22 & 0.39 \\
\hline $\operatorname{MERIS}\left(\mathrm{B}_{7}^{-1}-\mathrm{B}_{9}^{-1}\right) \times \mathrm{B}_{10}$ & 0.59 & 0.72 & 0.41 \\
\hline $\operatorname{Rrs}_{725} / \operatorname{Rrs}_{675}$ & 0.36 & 0.90 & 0.54 \\
\hline $\operatorname{Rrs}_{690}$ & 0.48 & 0.82 & 0.43 \\
\hline FLH & 0.22 & 1.00 & 0.53 \\
\hline
\end{tabular}

\section{CONCLUSIONS}

According to the principle of three-band model, a good linear relationship between the three-band model and chla concentration was built. And the in situ spectral data were averaged to the band range of MERIS so as to build the simulated three-band model of MERIS in this study. There was also a good linear correlation between MERIS threeband model and chla concentration, with the RMSE of $0.72 \mu \mathrm{g} \cdot \mathrm{L}^{-1}$. When three-band model was applied to retrieve chla concentration, we could get good retrieval precision even if the chla concentration is lower than $10 \mu \mathrm{g} \cdot \mathrm{L}^{-1}$. On the whole, the three-band model could be applied to retrieve chla concentration in Yantai coastal waters. And we could use MERIS data to retrieve and map chla concentration in Yantai coastal waters.

\section{ACKNOWLEDGEMENTS}

This research was supported by Chinese Natural Science Foundation projects (NSFC41271349), knowledge innovation project of Chinese academy of sciences (KZCX2-YW-Q07-04) and the science technology planning project of Shandong province (2007GG2QT06019). The authors thank all the crews for their assistance in sampling onboard. 


\section{REFERENCES}

[1] A. Morel and L. Prieur, "Analysis of variations in ocean color", Limnology and Oceanography, 22(4): 709-722, (1977).

[2] G. Dall'Olmo and A.A. Gitelson, "Effect of bio-optical parameter variability on the remote estimation of chlorophyll-a concentration in turbid productive waters: experimental results", Applied Optics, 44(3): 412422, (2005).

[3] H.J. Gons, "Optical Teledetection of Chlorophyll a in Turbid Inland Waters", Environmental Science \& Technology, 33(7): 1127-1132, (1999).

[4] J.F.R. Gower, R. Doerffer, and G.A. Borstad, "Interpretation of the $685 \mathrm{~nm}$ peak in water-leaving radiance spectra in terms of fluorescence, absorption and scattering, and its observation by MERIS", International Journal of Remote Sensing, 20(9): 1771-1786, (1999).

[5] A. Vasilkov and O. Kopelevich, "Reasons for the appearance of the maximum near $700 \mathrm{~nm}$ in the radiance spectrum emitted by the ocean layer", Oceanology, 22(5): 697-701, (1982).

[6] X. Xing, D. Zhao et al., "In situ determination of sun-induced chlorophyll a fluorescence quantum yield in the North China Sea", International Journal of Remote Sensing, 29(3): 851-865, (2008).

[7] Q. Xing, C. Chen et al., "Estimation of chlorophyll-a concentrations in the Pearl River Estuary using in-situ hyperspectral data: A case study.", Marine Technology Society Journal, 42(4): 22-27, (2008).

[8] K.G. Ruddick, H.J. Gons et al., "Optical remote sensing of chlorophyll a in case 2 waters by use of an adaptive two-band algorithm with optimal error properties", Applied Optics, 40(21): 3575, (2001).

[9] A.A. Gitelson, J.F. Schalles, and C.M. Hladik, "Remote chlorophyll-a retrieval in turbid, productive estuaries: Chesapeake Bay case study", Remote Sensing of Environment, 109(4): 464-472,(2007).

[10] A.A. Gitelson, G. Dall'Olmo et al., "A simple semi-analytical model for remote estimation of chlorophyll-a in turbid waters: Validation", Remote Sensing of Environment, 110(112): 3582-3593,(2008).

[11] T.R. Fisher, L. Harding et al., "Phytoplankton, nutrients, and turbidity in the Chesapeake, Delaware, and Hudson estuaries", Estuarine, Coastal and Shelf Science, 27(1): 61-93, (1988).

[12] H.R. Gordon, O.B. Brown, and M.M. Jacobs, "Computed relationships between the inherent and apparent optical properties of a flat homogeneous ocean", Applied Optics, 14(2): 417-427, (1975).

[13] G. Dall'Olmo, A.A. Gitelson, and D.C. Rundquist, "Towards a unified approach for remote estimation of chlorophyll-a in both terrestrial vegetation and turbid productive waters", Geophysical Research Letters, 30(18): 1938-1941, (2003).

[14] A.A. Gitelson, A. Vina et al., "Remote estimation of canopy chlorophyll content in crops", Geophysical Research Letters, 32(8): 403-406, (2005).

[15] A.A. Gitelson, A. Vina et al., "Remote estimation of leaf area index and green leaf biomass in maize canopies", Geophysical Research Letters, 30(5):1248-1251, (2003).

[16] P.V. Zimba and A. Gitelson, "Remote estimation of chlorophyll concentration in hyper-eutrophic aquatic systems: Model tuning and accuracy optimization", Aquaculture, 256(1-4):. 272-286, (2006).

[17] A.A. Gitelson, G. Dall'Olmo et al., "A simple semi-analytical model for remote estimation of chlorophyll-a in turbid waters: Validation", Remote Sensing of Environment, 112(9): 3582-3593, (2008).

[18] F.E. Hoge, C.W. Wright, and R.N. Swift, "Radiance-ratio algorithm wavelengths for remote oceanic chlorophyll determination", Applied Optics, 26(11): 2082-2094, (1987).

[19] D. Pierson and N. Strömbäck, "A modelling approach to evaluate preliminary remote sensing algorithms: Use of water quality data from Swedish great lakes", Geophysica, 36(1-2): 177-202, (2000).

[20] J. Seppala, P. Ylostalo, and H. Kuosa, "Spectral absorption and fluorescence characteristics of phytoplankton in different size fractions across a salinity gradient in the Baltic Sea", International Journal of Remote Sensing, 26(2): 387-414, (2005).

[21] F.E. Hoge, P.E. Lyon et al., "Validation of Terra-MODIS phytoplankton chlorophyll fluorescence line height. I. Initial airborne lidar results", Applied Optics, 42(15): 2767-2771, (2003). 